VOL. 74 (2006) [153-154]

\title{
On closures of finite permutation groups
}

\author{
JING XU
}

In this thesis we investigate the properties of $k$ closures of certain finite permutation groups. Given a permutation group of $G$ on a finite set $\Omega$, for $k \geqslant 1$ the $k$-closure $G^{(k)}$ of $G$ is the largest subgroup of $\operatorname{Sym}(\Omega)$ with the same orbits as $G$ on the set $\Omega^{k}$ of $k$-tuples from $\Omega$.

The first problem in this thesis is to study the 3-closures of affine permutation groups. In 1992, Praeger and Saxl Showed if $G$ is a finite primitive group and $k \geqslant 2$ then either $G^{(k)}$ and $G$ have the same socle or $\left(G^{(k)}, G\right)$ is known. In the case where the socle of $G$ is an elementary Abelian group, so that $G$ is a primitive group of affine transformations of a finite vector space, the fact that $G^{(k)}$ has the same socle as $G$ gives little information about the relative sizes of the two groups $G$ and $G^{(k)}$. In this thesis we use Aschbacher's Theorem for subgroups of finite general linear groups to show that, if $G \leqslant A G L(d, p)$ is an affine permutation group which is not 3-transitive, then for any point $\alpha \in \Omega, G_{\alpha}$ and $\left(G^{(3)} \cap A G L(d, p)\right)_{\alpha}$ lie in the same Aschbacker class. Our results rely on a detailed analysis of the 2-closures of subgroups of general linear groups acting on non-zero vectors and are independent of the finite simple group classification. In addition, modifying the work of Praeger and Saxl in [1], we are able to give an explicit list of affine primitive permutation groups $G$ for which $G^{(3)}$ is not affine.

The second research problem is to give a partial positive answer to the so-called Polycirculant Conjecture, which states that every transitive 2-closed permutation group contains a semiregular element, that is, a permutation whose cycles all have the same length. This would imply that every vertex-transitive graph has a semiregular automorphism. In this thesis we make substantial progress on the Polycirculant Conjecture by proving that every vertex-transitive, locally-quasiprimitive graph has a semiregualr automorphism. The main ingredient of the proof is the determination of all biquasiprimitive permutation groups with no semiregular elements.

Received 26th April, 2006

Thesis submitted to the University of Western Australia December, 2005. Degree approved, March 2006. Supervisors: Professor Cheryl Praeger, A/Professor Cai Heng Li and Dr Michael Giudici.

Copyright Clearance Centre, Inc. Serial-fee code: 0004-9727/06 \$A2.00+0.00. 


\section{REFERENCES}

[1] C.E. Praeger and J. Saxl, 'Closures of finite primitive permutation groups', Bull. London Math. Soc 24 (1992), 251-258.

School of Mathematical Sciences

Peking University

Beijing 100871

China

e-mail xujing@math.pku.edu.cn 